\title{
Diagnostic Imaging of Gastrointestinal Tumours in Dogs and Cats: A Review
}

\author{
${ }^{1}$ Francesco Simeoni, ${ }^{1}$ Francesca Del Signore, ${ }^{2}$ Rossella Terragni, \\ ${ }^{1}$ Roberto Tamburro, ${ }^{1}$ Giovanni Aste and ${ }^{1}$ Massimo Vignoli \\ ${ }^{1}$ Faculty of Veterinary Medicine, University of Teramo, Piano D'Accio, Teramo, Italy \\ ${ }^{2}$ Pet Care Veterinary Clinic, Bologna, Italy
}

\author{
Article history \\ Received: 11-12-2019 \\ Revised: 20-02-2020 \\ Accepted: 09-04-2020 \\ Corresponding Author: \\ Rossella Terragni \\ Pet Care Veterinary Clinic, \\ Bologna, Italy \\ Email: terragni.rossella@gmail.co
}

\begin{abstract}
Gastrointestinal tract tumours in dogs and cats are characterized by non-specific clinical presentation and laboratory abnormalities, but require fast identification, characterization and staging. Imaging techniques play a key role in the diagnostic process of these diseases in routine veterinary practice. Survey and contrast radiography may indicate primary or secondary signs of gastric or intestinal neoplasia such as a wall mass, functional alterations and stenosis points, but they have low sensibility and limited diagnostic value. Ultrasound allows to perform a detailed study of gastrointestinal wall's layers and thickness and adjacent organs but requires adequate acoustic windows and expert operators. Computed tomography is a reference technique both in human and veterinary oncology for the identification of primary neoplasms and for metastases searching thanks to the possibility to scan the whole body and the use of intravenous iodinated contrast medium for vascular assessment. Specific intraluminal distension techniques such as Helical-Hydro Computed Tomography and Computed Tomography Colonography can improve respectively stomach and colon associated masses. To date, Endoscopic Ultrasonography and Magnetic Resonance, despite their proven value in human medicine, lack of literatures that support their usefulness in the diagnosis and staging of gastrointestinal neoplasms in veterinary medicine and are not widely used in these pathologies. The purpose of this work is to review the literature on imaging modalities applied in gastrointestinal tumours diagnosis in dogs and cats, highlighting advantages and limitations of each technique in order to choose the proper imaging procedure in everyday clinical practice.
\end{abstract}

Keywords: Dog, Cat, Diagnostic Imaging, Gastrointestinal Tract, Neoplasia

\section{Introduction}

Gastrointestinal (GI) tract neoplasia is uncommon in both dogs and cats, but due to the non-specific clinical presentation and laboratory abnormalities, these tumours require a rapid diagnosis and characterization (Withrow, 2007). Adenocarcinoma (AC) is the most common primary GI malignancy in dogs (Terragni et al., 2014a), while lymphoma is the most common in cats (Gieger, 2011; Willard, 2012). Other malignancies described in the canine and feline GI tract include mast cell tumour, Gastrointestinal Stromal Tumour (GIST), leiomyosarcoma, fibrosarcoma, poorly differentiated sarcoma and carcinoid. Benign tumour has been reported to occur both in dogs and cats GI tract which includes polyps and leiomyomas (Willard, 2012).

Clinical signs depend on which portion of the GI tract is involved and usually include chronic vomiting and/or diarrhea, gastro-intestinal blood loss (hematemesis, melena and/or hematochezia) with weight loss that could result from anorexia, malabsorption and/or maldigestion, loss of protein or generalized tumour cachexia. Laboratory abnormalities may include panhypoproteinemia, microcytic hypochromic anemia related to chronic gastro-intestinal blood loss and malabsorption (Willard, 2012; Terragni et al., 2014b; Gianella et al., 2017).

Adenocarcinoma is the most common tumour type of the canine stomach, counting the $70-80 \%$ of gastric neoplasias (Swann and Holt, 2002) and the second most common intestinal tumour in the cat (Willard, 2012); moreover, it is the most common primary neoplasm of dog's rectum and cat's colon (Slawienski et al., 1997). Several studies reported a breed-related incidence of AC in German shepherd, Chow-chow, Belgian shepherd, Collies and Staffordshire bull terrier dogs and in Siamese 
cats (Scanziani et al., 1991; Penninck, 1998; Bilek and Hirt, 2007; Lubbes et al., 2009), thus implying a genetic component predisposition. Regarding the incidence of age and gender, older dogs are the most affected even if the age range is large (from 1 to 14 years of age); males are more frequently affected than females (Paoloni et al., 2002). It is reported that more than $40 \%$ of the dogs and more than $50 \%$ of the cats have detectable metastases at the time of the diagnosis (Rivers et al., 1997; Paoloni et al., 2002). At one institution, regional lymph nodes involvement was present in 14/17 of the dogs with gastric AC (Penninck, 1998). Common sites for metastases are, liver, mesentery, omentum and peritoneal surface as miliary lesions (Rivers et al., 1997; Crawshaw et al., 1998; Paoloni et al., 2002); other less reported sites are lungs, spleen, kidneys, pancreas, bladder, testis and urethra (Paoloni et al., 2002). Survival time in dogs with confirmed metastasis is approximately 3 months and 1 year in only $20 \%$ of patients (Crawshaw et al., 1998).

Lymphoma is the most common GI-related malignancy in the cat, representing $30 \%$ of all feline neoplasms; moreover, $70 \%$ of cats with lymphoma have GI involvement: In this species, the small intestine is the most commonly affected site and is also the most frequent tumour of the gastric wall (Willard, 2012; Paulin et al., 2018). The Siamese breed and the older age (between 10 and 14 years in particular) are correlated to an increased risk (Griffin, 2019). Lymphoma is less common in dogs than in cats; furthermore, Alimentary Lymphoma (AL) represents only $7 \%$ of all canine lymphoma (Gieger, 2011). The GI tract can be diffusely or focal infiltrated by neoplastic lymphocytes, with or without involvement of other abdominal organs such as liver, spleen and mesenteric lymph nodes (Gieger, 2011; Paulin et al., 2018). According to different classifications, AL can be divided into several subtypes depending on the histological characteristics: Low, intermediate or high histological grade; small or large cell size; $\mathrm{T}$ or B infiltrated neoplastic lymphocytes (Paulin et al., 2018). The main types of feline AL include "mucosal lymphoma" which is usually a Low-Grade, small T-cell forms of Alimentary Lymphoma (LGAL) and "transmural lymphomas", commonly a High-Grade, small or large B- or T-cell type of Alimentary Lymphomas (HGAL) (Paulin et al., 2018). At one institution, feline AL was equally divided among those types (Lingard et al., 2009), but in another study, the small cell lymphoma occurred three times more often than the large cell one (Fondacaro et al., 1999). Large Granular Lymphocytic Lymphoma (LGLL) is the less common, but the most aggressive, subtype of AL characterized by the presence of natural killer $\mathrm{T}$ lymphocytes with intracytoplasmic granules (Gieger, 2011; Paulin et al., 2018). Clinically, these types of AL are distinct entities with different clinical findings, therapies and outcomes.
Mesenchymal tumours such as leiomyomas and leiomyosarcomas have classically been the connective tissue tumour diagnosed in the canine and feline GI tract, but immunohistochemistry has allowed pathologists to distinguish Gastrointestinal Stromal Tumours (GIST) originating from the interstitial cells of Cajal (Russell et al., 2007). These neoplasms may be indistinguishable morphologically from smooth muscle tumours or other spindle cell tumours and routine histologic staining and immunohistochemistry are required in order to differentiate these neoplasm types, with GISTs being characterized by the expression of the protein CD117 and CD34 (Willard, 2012). Colon and cecum seem to be the most common localizations of GIST, whereas stomach is more often affected by leiomyomas and leiomyosarcomas (Hobbs et al., 2015).

Mast Cell Tumour (MCT) of the GI tract is the third most common intestinal tumour in cats (Antognoni et al., 2003). It may occur in any section of the GI tract with no association with cutaneous involvement. It is a highly malignant tumour with a high rate of metastasis and clinical pathology findings tend to be nonspecific, but abdominal effusion with mast cells may occur. Mastocytosis is infrequently seen (as opposed to splenic mastocytosis, which is more common) and eosinophilia may be detected in some patients (Willard, 2012).

\section{Imaging Modalities and Techniques}

As previously mentioned, neoplasms of the GI tract can cause a specific clinical sign that may be the result of structural alteration of the GI wall with or without compromising functionality (motility and digestion/absorption) and, in most cases, the first diagnostic line to investigate these clinical manifestations includes, in addition to laboratory tests, the use of imaging techniques like survey radiography and ultrasonography.

Survey radiographs and contrast radiography have been considered the principal diagnostic imaging techniques used to examine the GI tract for many years. Contrast radiography studies are useful for the visualization of intraluminal masses, stenosis point or motility anomalies but is a potentially time-consuming procedure, often requiring multiple exposures and expert operator for an optimal interpretation. Even under optimal conditions, the sensitivity and specificity of radiography for many GI conditions are limited (Lamb and Grierson, 1999).

Ultrasonography (US) of the canine and feline abdomen is a non-invasive, widely used technique that allows to assess the GI tract evaluating wall thickness and layers, motility and lumen content, getting useful information of adjacent structures such as lymph nodes and peritoneum. To perform a complete US assessment of the GI tract, different types of transducers should be used: A microconvex transducer $(5-10 \mathrm{MHz}$ of frequency range) allows evaluation of all GI tract in both dogs and 
cats; in large breeds dogs, a lower frequency (3.5-5 MHz) may be necessary to visualized the deepest part of the abdomen; a high frequency linear transducer (7-15 MHz) allows detailed images acquisition of wall's layers of all the GI tract in small breeds dogs and cats and of the most superficial portion in larger dogs. (Terragni et al., 2014b). Whole wall thickness measurements are taken from the inner mucosal interface to the outer aspect of the serosa. The motility of the GI tract can be assessed by counting peristaltic waves that are usually 3-5 min in both healthy dogs and cats (Larson and Biller, 2009). Peristaltic activity can routinely be observed in the stomach and small bowel but not in the colon (Penninck, 1998). Ultrasonography proved through the years to be a sensitive and versatile modality for morphological lesions affecting the GI tract and in many cases is able to confirm or rule out any suspected diseases, offering the practical advantages of being less time-consuming than contrast radiography and not involving the use of ionizing radiation. Nevertheless, gas may inhibit complete visualization of the GI wall and lumen and optimal GI US requires relatively highresolution equipment and an expert operator (Lamb and Grierson, 1999; Larson and Biller, 2009). However, abdominal radiograph and US are complementary imaging modalities and each adds useful information.

The technique of Endoscopic Ultrasonography (EUS) is widespread in human medicine, especially to investigate the lumen, the wall and the organs close to the GI tract. In veterinary medicine, current applications for EUS in GI tract assessment include an examination of the esophagus, gastric wall, duodenum and jejunum, as well as detection of regional lymphadenomegaly and the possibility of performing fine-needle aspiration biopsy of the GI wall, adjacent organs and extraluminal structures (Gaschen et al., 2007). Other potential applications in dogs and cats include tumours staging and intrapelvic US (Gaschen et al., 2003). However, in veterinary medicine, only few publications are present for limited use (Lamb and Grierson, 1999; Gaschen et al., 2003; 2007).

Computed Tomography (CT) scanners allow whole body images acquisition with optimal delineation of primary masses, involved lymph nodes and others organs metastases in veterinary oncology (Fukushima et al., 2012; Terragni et al., 2012; Fife et al., 2004; Kutara et al., 2014; Fukushima et al., 2016; Forrest, 2016; Carloni et al., 2019); but, only sporadic studies have reported the CT imaging characteristics of GI's tumours in veterinary medicine (Hoey et al., 2012; Terragni et al., 2012; Tanaka et al., 2019; Yoon et al., 2019).

\section{Radiography}

\section{Stomach}

For a specific radiographic examination of the stomach, four projections are needed: Ventrodorsal, dorsoventral, left and right lateral. Using these projections, gas within the lumen can be manipulated to visualize all the gastric regions. In normal condition, the axis of the stomach is parallel to the ribs on the lateral projection. In canine stomach on the ventrodorsal view, the canine axis is perpendicular to the spine, with fundus and most of the body located on the left side, while the pyloric region is on the right; in the feline species, the axis is parallel to the spine, with the pylorus located at or near the midline and is more acutely angled with the stomach (Terragni et al., 2014b). The normal gastric wall appears smooth and uniform and is a few mm thick when fully distended. To increase the sensitivity of the radiographic study in identifying gastric masses, the use of intraluminal contrast agents may be necessary. Several contrast techniques have been described through the years: Positive-contrast gastrography using barium or iodinated contrast medium; pneumogastrography using negative-contrast created by filled $\mathrm{CO}_{2}$ or air into the gastric lumen; double-contrast gastrography using a positive contrast followed by gas; low-volume gastrography (Terragni et al., 2014b). Each type of contrast radiography should always be preceded by survey radiographs and any patient that would perform a contrast study must be fasted for at least $12 \mathrm{~h}$. After barium or iodinate administration, each projection is repeated at $0,15,30 \mathrm{~min}$ and 1,2 and 3 hours to follow the progression of the contrast medium in and out of the stomach (Wallack, 2003). Also, the motility of the organ is contextually evaluated since the times of gastric emptying have been established in both dogs (30120 min) (Miyabayashi and Morgan, 1986) and cats (15-30 min) (Morgan, 1981).

On survey radiographs, findings of gastric lesions may include masses that protrude into the lumen, but, if the stomach is not filled with air and or too much food is present, the lesion may not be detected (Terragni et al., 2014b; Valentini et al., 2013). On contrast radiography, gastric neoplasia could be better visualized as a filling defect within the contrast medium outlining the lesion protruding into the lumen. In some cases, large stenotic masses may lead to outflow obstruction (Teshima et al., 2013).

Gastric AC usually presents as diffuse thickening of the gastric wall or less frequently, shows a focal wall thickening at the level of lesser curvature and pylorus. Gastric leiomyoma and leiomyosarcoma share similar features: Both tumours tend to present as mass lesion that extends asymmetrically from the gastric wall and does not affect the lumen or, less frequently, they appear as a distinct mass within the stomach lumen. Lymphoma may also present as a focal mass that extends out from the gastric wall and does not affect the lumen (Gualtieri et al., 1999; Willard, 2012; Terragni et al., 2014b). Secondary signs of a gastric tumour include the presence of a gastric ulcer, an abnormal pattern of rugal folds, poor 
local or diffuse gastric distension, lack of peristalsis and delayed gastric emptying. However, radiology of the stomach is not sensible for the identification of intramural lesions (Terragni et al., 2014b).

\section{Intestine}

Survey radiography and contrast radiography are useful diagnostic imaging methods used to evaluate the canine intestine The animal should ideally fast for 12 hours before the radiographic examination and if at the time of the first survey radiography, too much fecal material is present in the colon, an enema may also be necessary. A standard right lateral and ventrodorsal projection of the abdomen are often used for the assessment of both small and large bowel. A moderate amount of gas is physiologically present in the small bowel of fasted dogs but is considered pathological in cats; instead, the colon may present moderate dilation by gas and feces in healthy dogs and cats. In left lateral recumbency, gas from the stomach often passes into the proximal duodenum and this is helpful if duodenal pathology such as neoplasia or foreign body is suspected (Paoloni et al., 2002). No accurate specific measurement for the normal diameter of canine and feline bowel exist, because of the variation in dogs body size and the magnification effects of radiography (Riedesel, 2017).

Intestinal neoplasia may not create radiographic abnormalities, especially on survey radiographs. Possible changes include a soft tissue mass of variable size with possible partial to complete obstruction resulting in bowel dilation (Paoloni et al., 2002; Gieger, 2011; Terragni et al., 2014b). Neoplastic cellular infiltration within the intestinal wall causes an increased wall thickness which, however, cannot be determined reliably from survey radiographs but can be evaluated with positive contrast studies. Secondarily, bowel motility may be altered, resulting in a change in bowel diameter. Some neoplasm like $\mathrm{AC}$ and $\mathrm{AL}$ may appear as a protruding or annular stenotic mass causing signs of total or partial obstruction, visible on survey radiography as a bowel dilation or accumulation of radiopaque foreign material ("gravel sign") proximal to the lesion (Paoloni et al., 2002; Gieger, 2011). There are no unique survey radiographic findings in intestinal tumour: Wall's masses may cause smoothly rounded lumen protrusions or deformation of the serosal contour and if the mural mass surrounds the lumen, that becomes enlarged and irregularly shaped (Riedesel, 2017). Contrast radiography provides a better definition in the detection of these lesions than survey radiographs, outlining more precisely the narrowing of the lumen at the tumour site (Paoloni et al., 2002). The wall layers cannot be assessed on radiography, but only on US.

\section{Ultrasound}

\section{Stomach}

Ultrasonographic evaluation of the stomach includes several advantages because of the non-invasive nature of the examination, other associated structures concomitant assessment like regional lymph nodes for a better staging in dogs and cats with gastric masses and the possibility to perform studies on conscious or only mildly sedated patients. Every patient undergoing US exam should be fasted for at least $12 \mathrm{~h}$ (Penninck, 1998; Terragni et al., 2014b), since the food and gas contained in the stomach will not allow a correct visualization of the gastric wall; moreover, for a better evaluation of this structure, it's possible to administer some water at $10-15 \mathrm{~mL} / \mathrm{kg}$ which will enhance wall's layers definition with optimal distention of the stomach, overcoming gas artifacts that may be present also in fasted animals (Penninck, 2002; Terragni et al., 2014b). Right lateral recumbency is useful for pyloric examination and left lateral for the fundus. In the empty stomach, prominent rugal folds are visible, especially in the cat, where they resemble a spoked wheel (Larson and Biller, 2009). Normal gastric wall thickness ranges from 3 to $5 \mathrm{~mm}$ (Penninck, 1998); when gastric wall measurements are collected, it is important to measure the wall between rugal folds to avoid artifactual increase in thickness, especially in the cat where folds are prominent and the distinction between wall and lumen is not always simple (Larson and Biller, 2009). The normal gastric wall consists in five layers: the inner mucosal-luminal interface (hyperechoic), the mucosal layer (thick and hypoechoic), the submucosal layer (hyperechoic and thin), the muscle layer (hypoechoic and thin) and the outer serosal surface (hyperechoic and thin) (Penninck, 1998; Newell et al., 1999). The major features of gastric neoplasia include focal thickening with loss of definition of normal wall's layers, with a higher sensitivity than radiography (Seiler and Maï, 2009). The thickening of the gastric wall can be generalized or localized, symmetrical or asymmetrical and associated or not with significant loss of motility (Terragni et al., 2014b).

The AC in dogs typically shows an asymmetrical, moderate to severe, heterogeneous gastric wall thickening. The loss of the normal wall's layers may be replaced by three alternating bands that appear as poorly defined, echogenic lining on the innermost and/or outermost portions of the gastric wall separated by a more echoic central zone; this particular finding is defined "pseudolayering" (Penninck, 1998, Terragni et al., $2014 b)$. This alteration is typical of gastric AC and reflects his histopathological structure (Penninck, 1998). Adenocarcinoma may often spread to regional lymph nodes (Penninck, 1998; Paoloni et al., 2002; Willard, 2012) and, when it happens, regional lymph nodes may 
present rounded with poorly defined echoic rim and a highly defined echoic center, also reported as target appearance (Penninck, 1998). In cats, gastric AC is extremely rare and only very few cases have been reported through the years (Gualtieri et al., 1999); however, the US characteristics of these gastric tumours have never been described in this species.

Canine and feline gastric lymphoma can show different presentations. Usually, the US appearance is described as a circumferential, transmural thickening associated with diffuse loss of normal wall layering and reduced wall echogenicity (Fig. 1A), associated with localized decreased motility and moderate regional lymphadenopathy (Penninck et al., 1994; Penninck, 2002). Another report described a gastric lymphoma as a focal hypoechoic eccentric mass (Seiler and Maï, 2009).

The most frequent location of leiomyoma and leiomyosarcoma is the pyloric region, with submucosal hypoechoic masses arising from the muscular layer as the most frequent ultrasonographic appearance. Leiomyomas usually appear as homogeneous, small, sessile, echoic masses in dogs (Myers and Penninck, 1994), while leiomyosarcomas are more often large, complex masses with an irregular central cavity (representing hemorrhage, necrosis or cystic degeneration) (Seiler and Maï, 2009); Leiomyosarcomas can ulcerate leading to wall perforation (Myers and Penninck, 1994).

Ultrasonographic features of malignant histiocytosis, malignant fibrous histiocytoma and histiocytic sarcoma have been sporadically reported (Kaser-Hotz et al., 1996; Cruz-Arámbulo et al., 2004) and other tumours, like carcinoid, neurilemmoma, nerve-sheath tumour, mast cell tumour and hemangiosarcoma, tend to appear as poorly defined echoic masses or as a focal thickening with loss of layering. No specific US features for these tumours have been described. Gastric wall thickening corresponding to wall edema and luminal fluid accumulation associated with reduced gastric motility may be observed in animals with large lesions, with ulcers as possible complications reported in the literature (Terragni et al., 2014b).

\section{Intestine}

Ultrasonography is useful in assessing intestinal uniformity in diameter, wall thickness and layers, luminal contents and peristalsis (Larson and Biller, 2009; Gaschen, 2011). A complete examination of the small intestine is performed by scanning the abdominal cavity in sagittal and transverse planes, visualizing the duodenum, the jejunum and the ileum in each of their portions. The colon may be scanned in its entirety, including the junction of small intestine with the colon in cats or cecum in dogs. If the patient is fasted for at least $12 \mathrm{~h}$, feces and gas may fill only the colon, but they can be present also in the small intestine, creating reverberation artifacts and acoustic shadowing making wall measurements difficult (Penninck, 1998;
Larson and Billler 2009). In the small intestine, the same layering of the stomach is observed (see above); colonic wall has a layered appearance like the small intestine but is thinner with less obvious layering; it can be easily identified because of its location and striking acoustic shadow and reverberation artifacts (Nyland et al., 2016). Normal small bowel wall thickness ranges approximately from 2 to $5 \mathrm{~mm}$; large bowel thickness, instead, ranges from 1 to 2,5 $\mathrm{mm}$ (Riedesel, 2017). The duodenal wall tends to be slightly more prominent than the remaining bowel in dogs, while in the cats, the ileum appears to be the thickest segment. With a highresolution probe, Peyer's patch (aggregated lymphoid follicles) may occasionally be seen in the duodenal portion and may be mistaken for ulcerative lesions (Penninck, 1998).

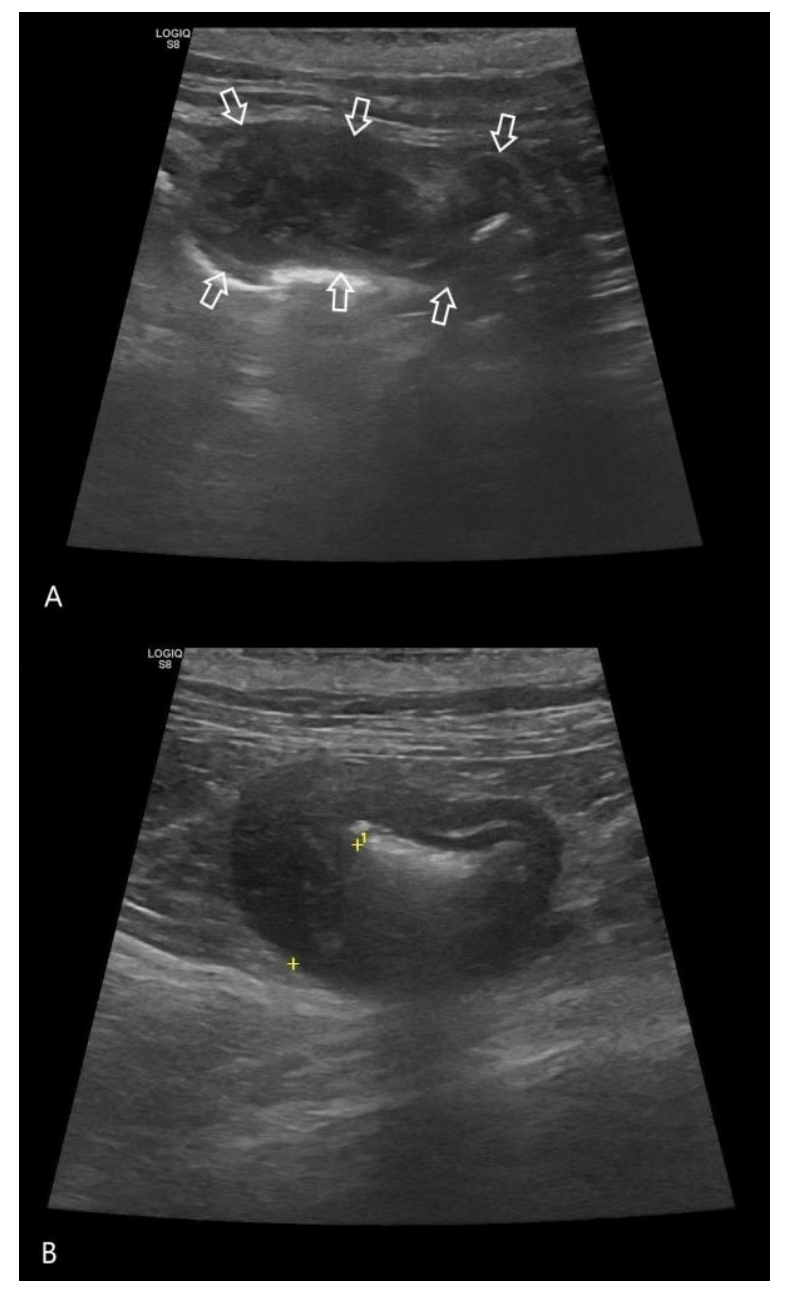

Fig. 1: Ultrasonographic images of a cat with gastric and jejunal high-grade lymphoma: The patient had multifocal hypoechoic transmural lesions with wall thickening and complete loss of layering (white arrows) in the stomach (A) and also a focal hypoechoic transmural thickening with loss of wall layering (between the cursors) in the jejunum (B) 
Intestinal neoplasms typically occur with a focal or multifocal concentric or eccentric, wall thickening with loss of wall layering. However, US findings in course of inflammatory diseases of the GI tract can be very similar to those of neoplastic infiltration, showing diffuse or multifocal mild wall thickening, loss of definition of wall layers and mesenteric lymphadenopathy. Since neoplasms and GI inflammation can overlap, parameters such as degree of thickening, loss of layering, symmetry and extension of the lesions are considered: As general rule, the more dramatic the alterations, the greater the suspicion of neoplasia (Penninck et al., 2003; Larson and Biller, 2009). However, in many cases, inflammation cause mild or none US alterations (Larson and Biller, 2009). Penninck et al. (2003) reported that dogs with neoplastic wall infiltration have a median wall thickness statistically greater than that of inflammatory disease (median: $15 \mathrm{~mm}$ vs $6 \mathrm{~mm}$, respectively) and that neoplastic involvement of the small bowel is more often focal than diffuse, which is more common in inflammation. Furthermore, the authors reported that loss of wall layering is 50-times more associated with the diagnosis of neoplasia than of nonspecific inflammation (Penninck et al., 2003). Carcinoma, lymphoma, leiomyoma and leiomyosarcoma are the most common intestinal wall tumours in dogs (Gaschen, 2011). One study has described the sonographic findings of an ileal hemangioma in a dog (Aita et al., 2010). In cats, AL, AC and mast cell tumour are the most common intestinal malignancy. Visceral hemangiosarcoma of the small bowel and colon has also been reported (Culp et al., 2008).

Intestinal AC in dogs and cats sonographically appear as a severe wall thickening with complete loss of wall layers and regional lymph nodes involvement (Willard, 2012). These findings are similar to that of HGAL; however, the distribution of carcinomas is usually solitary whereas AL can be focal, multifocal, or diffuse (Penninck, 1998; Gieger, 2011). Furthermore, intestinal AC often cause intraluminal stenosis and mechanical ileus, which is less frequent in lymphomas (Penninck, 1998; Paoloni et al., 2002). In a retrospective study of 21 dogs with intestinal AC, data about localization, length, wall thickness, echogenicity, regional motility, layering, regional lymphadenopathy and fluid accumulation proximal to the lesion site were sonographically evaluated: In 15 dogs the tumour was identified in the small bowel (6 had confirmed jejunal lesions) and in the colon for the other 6 dogs $(2$ colonic and 2 colorectal confirmed lesions) and all lesions had transmural thickening ranged from 7 to $17 \mathrm{~mm}$ (median $12 \mathrm{~mm}$ ) with complete loss of wall layering. Lesion length ranged from 23 to $63 \mathrm{~mm}$ (median $40 \mathrm{~mm}$ ). For what concerned the echogenicity, most intestinal lesions were hypoechoic with irregular edges at the lumen interface.
Fluid accumulation proximal to the lesion site was identified in 17 of 21 dogs. In 13 of 17 dogs the fluid accumulation was considered moderate to severe and in 12 of 21 dogs had regional lymphadenopathy and/or carcinomatosis (Paoloni et al., 2002). Another study reported ultrasonographic findings of 5 cats with intestinal AC: In all 5 cats a mass was clinically palpable but radiographically an abdominal mass was detected in only 1 cat. The ultrasonographic features of the lesions are a segmental intestinal mural mass (5 of 5 cats) characterized by circumferential bowel wall thickening with transmural loss of normal wall layers; in 1 cat, the circumferential symmetric hypoechoic bowel wall thickening was similar to what is reported for segmental lymphoma. In the other 4 cats, the sonographic features of the thickened bowel wall varied, being mixed echogenicity and asymmetric in 3 cats and mixed echogenicity and symmetric in 1 (Rivers et al., 1997).

Alimentary lymphoma can appear with different features in the GI tract: It can occur as a focal or multifocal hypoechoic intestinal mass with transmural loss of wall layering, causing partial stenosis of the intestinal lumen or it may appear as a normal or increased intestinal wall thickness with preservation of intestinal layers (Penninck, 1998; Lingard et al., 2009; Gieger, 2011; Gaschen, 2011). Furthermore, it is the most common neoplastic cause of diffuse infiltration and wall thickening in cats that can appear similar to Inflammatory Bowel Disease (IBD) (Gaschen, 2011; Gianella et al., 2017). In cats, AL, as previously told, is the most commonly encountered form of lymphoma and the three main histological types, LGAL, HGAL and LGLL, have different diffusion, aggressiveness and prognosis. The ultrasonographic aspect seems to change with the different types of infiltrates (Gieger, 2011; Willard, 2012).

Ultrasonographic aspects of HGAL in cats may include a localized, hypoechoic, transmural intestinal thickening with loss of definition of wall's layers (Fig. 1B), hypomotility and abdominal lymphadenomegaly. Concurrent alteration of liver, with hyperechoic or hypoechoic parenchyma, pancreas and spleen, with diffuse echogenicity changes and/or nodular lesions, may also be present supporting the possible infiltration of these organs (Zwingenberger et al., 2010). For what concern LGAL, several authors reported the ultrasonographic characteristics of this lymphoma and the fact that it is, in most cases, clinically and histologically indistinguishable from IBD (Lingard et al., 2009; Zwingenberger et al., 2010; Daniaux et al., 2013). In one study on cats with LGAL, the most common ultrasonographic finding was normal or mild increased intestinal wall thickness (mean $4.3 \mathrm{~mm}$, range $3.4-5 \mathrm{~mm}$ ) with preservation of layering (Lingard et al., 2009). In a 
further study, 22/27 (81\%) cats with LGAL had evidence of intestinal thickening on ultrasound (Stein et al., 2010). Another study evaluating differences in ultrasound findings between cats with LGAL versus IBD reported that cats with LGAL have thickening of the muscularis layer 18 times more frequently than those with IBD. In addition, in the course of LGAL, lymphadenopathy is present in $47 \%$ of subjects and only in $17 \%$ of those with IBD; the combination of muscularis thickening and lymphadenopathy was present in $26 \%$ of LGAL whereas only 1 of 24 cats with IBD had both findings (Zwingenberger et al., 2010). Daniaux et al. (2013) reported that cats affected by LGAL and IBD a had an increased thickening of the muscolaris propria which was on average twice the size compared with healthy cats. Furthermore, the muscularis was the major contributor to significant overall bowel wall thickening in the duodenum and jejunum supporting that a muscularis to submucosa ratio $>1$ could be indicative of an abnormal bowel segment (Daniaux et al., 2013). In another study of 16 cats with LGAL, mesenteric lymphadenopathy was present on US in 12 cats, diffuse small intestinal wall thickening in 9, a focal intestinal mass in 1 and concurrent lymphoplasmacytic IBD was diagnosed in 4 cases (Lingard et al., 2009). In a recent study, enzyme Lactate Dehydrogenase (LDH) has been studied for differentiating between AL from a nonneoplastic disease such as IBD (Terragni et al., 2016). Sonographic findings in 71 cats included revealed: moderate to severe GI transmural thickening associated with loss of layering in the 14 cats with HGAL; mild increased of the muscularis layer with normal layering in 25 cats (14 LGAL, 73.7\%; 11 IBD, $28.9 \%$ ); normal wall thickening and layering in 5 cats with LGAL (26.3\%) and in 27 with IBD (71.1\%). Mesenteric lymph nodes enlargement was present in 32 cats, 25 with lymphoma $(75.8 \%)$ and 7 with IBD (18.4\%). Alimentary lymphoma is less common in dogs than in cats and LGAL is rare in this species (Gieger, 2011). One study reported that intestinal lymphoma may be present in bowel with normal or minimal alteration in wall thickness (highly variable with approximately equal numbers in the normal to mild, moderate and severe categories) and layering (20\% of the patient retained normal wall layering), suggesting that distortion of wall layering and thickness are poorly sensitive for the diagnosis of lymphoma also in dogs and 4 patients with histopathologically confirmed lymphoma showed no sonographic alterations (Frances et al., 2013). One study examined duodenal perfusion using Contrast-Enhanced
Ultrasonography (CEUS) in dogs with chronic inflammatory enteropathy ( $n=26$ dogs classified into: 16 Remission and 10 symptomatic based on clinical scores), intestinal lymphoma ( $n=7$ dogs) and a control group with GI signs but histopathologically normal duodenum $(n=$ 14) showing no significant differences in perfusion parameters between the lymphoma and inflammatory groups or the lymphoma and control groups (Nisa et al., 2019). Ultimately, the optimal approach to diagnose GI lymphoma varies among clinicians: Fine-needle aspiration and cytology of intestinal masses and enlarged mesenteric lymph nodes are relatively non-invasive and rapid diagnostic methods. However, the presence of inflammation and/or lymphoid reactivity may hinder a definitive diagnosis and histopathology of endoscopically or surgically obtained intestinal biopsy may be necessary to confirm the diagnosis (Gieger, 2011).

Intestinal smooth muscle tumours such as leiomyosarcomas originate intramurally and may bulge out of the serosa appearing as extraluminal masses. The masses often become very large and contain hypo/anechoic areas, representing central necrosis (Myers and Penninck, 1994). Leiomyomas tend to be small and appear as a focal intramural hypoechoic thickening with loss of wall layering (Myers and Penninck, 1994).

Only one retrospective cross-sectional study of 37 dogs described the ultrasonographic features of canine GISTs (19 dogs) compared with other spindle cell tumours (8 leiomyosarcomas, 6 leiomyomas and 4 no specified sarcomas). GISTs were significantly more likely to be associated with the cecum (8/8 of cecum lesions) and with abdominal effusion (58\% of GISTs) than other tumour types, but no ultrasonographic feature other than this was helpful in differentiating between GI spindle cell tumour types. The only tumour type reported without cavitated regions was leiomyoma (6/6) (Hobbs et al., 2015).

Intestinal MCT is rare and are more common in cats than in dogs. The sonographic features of intestinal MCT were reviewed by Laurenson et al. (2011) in one study of 14 cats (16 focal intestinal tumours and 1 diffuse submucosal infiltrate): The most common pattern was focal, hypoechoic wall thickening that was noncircumferential and eccentric (9/16 tumours) or circumferential, asymmetric and eccentric (5/16 tumours); 6/16 tumours had altered but not complete loss of wall layering and the most commonly affected layer on ultrasound examination was the muscularis propria. For what concerned the localization, $9 / 14$ of the cats had lesions in the jejunum or duodenum, $4 / 14$ were at the ileo-caeco-colic junction and 1/14 patient had a colonic mass. Enlarged abdominal lymph nodes were present in 9/14 cats ( 7 were due to metastatic disease) and concurrent small $\mathrm{T}$ cell lymphoma was present in $4 / 14$ cats (Laurenson et al., 2011). 


\section{Computed Tomography and Magnetic Resonance}

Computed Tomography is one of the most valuable imaging techniques for diagnosis and staging in veterinary oncology (Fukushima et al., 2012; Fife et al., 2004; Kutara et al., 2014; Fukushima et al., 2016; Forrest, 2016; Carloni et al., 2019). Dogs and cats usually undergo general anesthesia during CT examination, placed in sternal recumbency and a nonionic water soluble iodinated positive contrast medium, injected into a peripheral vein, is routinely used in order to perform vascular assessment (Forrest, 2016). The administration of water as a neutral contrast agent has been described as Helical-Hydro CT (HHCT) by Terragni et al. (2012) to improve visualization of the gastric wall by an optimal distension of the lumen in dogs and cats with gastric neoplasia. The procedure consist of the administration of $30 \mathrm{~mL} / \mathrm{kg}$ of water as a neutral luminal contrast medium followed by the intravenous injection of iodinated contrast medium, obtaining a uniform distension of the gastric lumen and enhancing visualization of the gastric wall obtaining uniform distension of the gastric lumen and enhancing visualization of the gastric wall. Computed Tomography Colonography (CTC) technique is described in human medicine as a useful method for the assessment of colonic wall: The use of $\mathrm{CO}_{2}$ as a negative intraluminal contrast medium may enhance visualization of colonic wall's lesions by distension of the lumen and contrast enhancement between the mucosal surface and the filled gas (McLaughlin et al., 2013). After colonic preparation, gas insufflation is performed using an endorectal positioned foley catheter and image acquisition is immediately performed once an adequate and stable rectal pressure is reached. Patients are scanned pre- and post-administration of intravenous iodinated contrast medium for an optimal visualization of wall's vascularization. No studies describing CTC technique are currently present in veterinary literatures, but this manuscript authors have already performed this method in one dog affected by a histologically confirmed AC of the descendent colon with good results in term of mass visualization and lumen distension (Fig. 2).
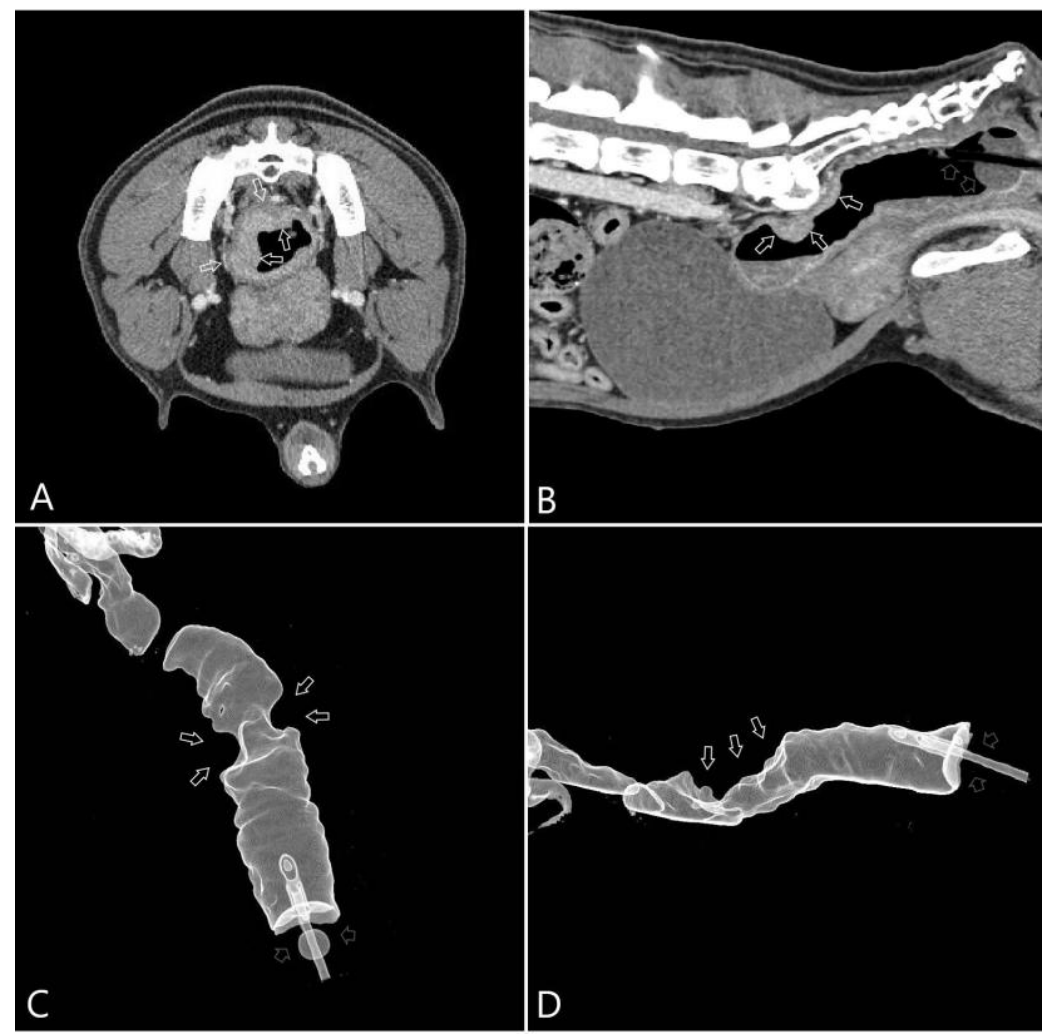

Fig. 2: Computed Tomography Colonography: Transversal (A) and sagittal (B) planes and volume rendering reconstruction of the gas filled into the colon lumen (C, D) of a dog affected by colonic AC. After the rectum and colon have been prepared by warm enemas, a Foley catheter was positioned (grey arrows). Then air was insufflated into the colonic lumen until adequate distension was obtained (A, B): A focal intraluminal protruding wall thickness with contrast uptake were visualized (white arrows). In postprocessing examination, the volume rendering of the gas filled into the descendent colon was reconstructed (C, D): This software elaboration shows clearly the circumferential filling defect present at the mural thickness point (white arrows) 
Only a few studies described CT usefulness in the assessment of GI neoplasms. One study described GI tract using pre and post-contrast CT through identification of GI segments, wall layers and thickness in 19 healthy dogs and 2 dogs with histologically confirmed GI neoplasia: In a Labrador retriever, a gastric Leiomyosarcoma has been described as a heterogeneously contrast-enhancing mural mass (13.7 $\mathrm{mm}$ with disruption, greater than healthy dogs range of 2-3,08 $\mathrm{mm}$ ) located within the dorsal portion of the fundic wall; a bulldog crossbreed with a circumferential focal thickening of the jejunum (wall thickness between 9 and $10.5 \mathrm{~mm}$, with healthy dogs range of 3,32-3,87 $\mathrm{mm}$ ) had histological diagnosis of AC. However, wall layers were visible only in $24 \%$ of all patients and there was no correlation between wall thickness in healthy dogs measured in CT and previous US standards (Hoey et al., 2012). The use of HHCT in 11 healthy animals ( 9 dogs and 2 cats) and 18 suspected gastric neoplasia (14 dogs and 4 cats) was assessed in veterinary medicine in one study (Terragni et al., 2012). In 18 patients with ultrasonographic suspect of gastric neoplasia, HHCT was able to confirm or exclude the presence of tumours identifying in detail: 8 Dogs with AC, described as focal, moderate gastric wall thickening with heterogeneous contrast enhancement and hyperattenuating mucosa; 1 dog with low-grade leiomyosarcoma that appeared as a mass with peripheral contrast enhancement filling almost all of the pyloric antrum; $1 \mathrm{dog}$ and 3 cats affected by lymphoma described as a moderate to severe gastric wall thickening with heterogeneous contrast enhancement of the lesion (Terragni et al., 2012). A recent retrospective case series conducted by Tanaka et al. (2019) reported dual-phase contrast CT findings in 16 dogs with confirmed gastric neoplasia. In addition to morphological and contrast uptake parameters on the site of primary gastric neoplasia, possible regional or peripheral involvement of the lymph nodes was also evaluated. The final diagnosis based on endoscopically or surgically obtained biopsy included $6 \mathrm{AC}, 5$ lymphomas, 3 inflammatory polyps and 2 leiomyomas. The major findings for the characterization of the tumour type are described below. A lower mean CT attenuation value for lymphoma than other gastric tumours on both postcontrast early and delayed phase was reported. For what concerned the lymph nodes pattern and involvement, lymphadenopathy was regional in $\mathrm{AC}$, widespread in lymphomas and not detected in leiomyomas. Also, lymph nodes measurements were reported to be larger in dogs with lymphomas than dogs with AC (Tanaka et al., 2019). Another recent case-report described the usefulness of dual-phase contrast CT in the intussusception diagnosis of the cecum into the ascending colon with a concurrent cecal mass with strong enhancement in arterial phase, in an 8 years old Labrador retriever. After surgical resection, histology confirmed a carcinoid tumour of the cecum that worked as a lead point for the intussusception (Yoon et al., 2019). This manuscript authors want to report a case of ileocaeco-colic AC with peritoneal carcinomatosis identified in one cat with CT (Fig. 3).

Magnetic Resonance Imaging (MRI) has proved to be a valuable technique in the assessment and of GI neoplasia in human medicine (Kim et al., 2017; Horvat et al., 2018; Borggreve et al., 2019); however, regarding the use of MRI in canine and feline GI tumours, no studies are present in veterinary literature.

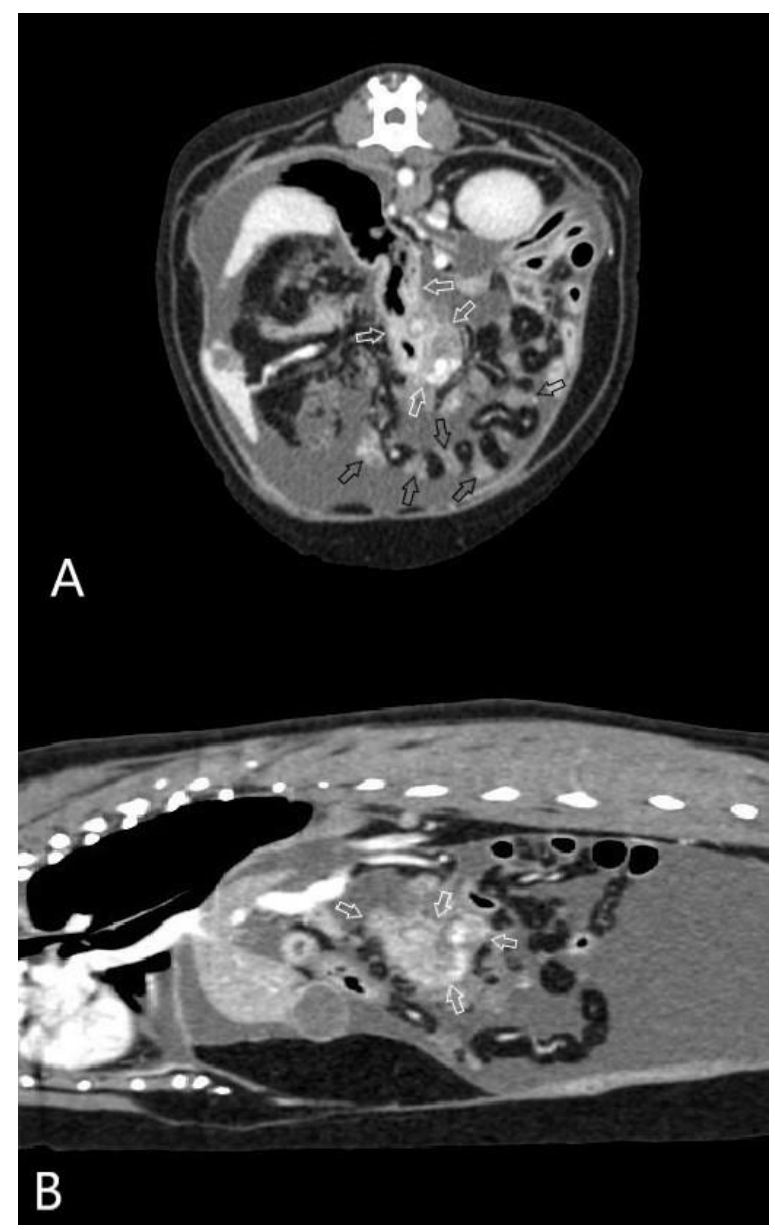

Fig. 3: Computed Tomography's images in transversal (A) and sagittal (B) planes of abdomen of a cat with histologically confirmed intestinal AC: The primary tumour was a heterogeneous mass involving the ileocaeco-colic junction (white arrows); carcinomatosis was present as postcontrast hyperdense nodular lesions in the peritoneum (black arrows) 


\section{Conclusion}

Diagnostic imaging has continued to be a crucial part in the assessment of GI tract's neoplastic diseases in everyday veterinary practice. Direct and contrast radiography can provide useful information despite the lack of sensitivity and reduced practicality. On the other hand, ultrasound appears to be a widely used and versatile technique able to identify abnormalities in wall thickness and layers. Also, CT has shown promising results in GI's neoplasia diagnosis and staging. In human medicine, EUS and MRI have become reference techniques in the diagnosis and staging of GI tract's tumours, but, to date, veterinary literature is incomplete or even absent. According to this manuscript authors, the widespread and proven use of CT, EUS and MRI in human GI neoplastic disease, should encourage further studies of these imaging techniques also in veterinary medicine.

\section{Acknowledgement}

The authors wish to thank the staff of Pet Care Veterinary clinic for the collaboration.

\section{Author's Contributions}

All authors equally contributed in this work.

\section{Ethics}

This article is original and contains unpublished material. The corresponding author confirms that all of the other authors have read and approved the manuscript and no ethical issues involved.

\section{References}

Aita, N., H. Iso and K. Uchida, 2010. Hemangioma of the ileum in a dog. J. Vet. Med. Sci., 72: 1071-1073. DOI: 10.1292/jvms.10-0017

Antognoni, M., A. Spaterna, E. Lepri, A. Fruganti and F. Laus, 2003. Characteristic clinical, haematological and histopathological findings in feline mastocytoma. Vet. Res. Commun., 27: 727-30. DOI: $10.1023 / \mathrm{b}$ :verc.0000014258.64713.ca

Bilek, A. and R.A. Hirt, 2007. Breed-associated increased the occurrence of gastric carcinoma in Chow-Chows. Wiener Tierarztliche Monatsschrift, 94: 71-79.

Borggreve, A.S., L. Goense, H.J.F. Brenkman, S. Mook and G.J. Meijer et al., 2019. Imaging strategies in the management of gastric cancer: Current role and future potential of MRI. British J. Radiol., 92: 20181044-20181044. DOI: $10.1259 /$ bjr. 20181044
Carloni, A., R. Terragni, A.M. Morselli-Labate, M. Paninarova and J. Graham et al., 2019. Prevalence, distribution and clinical characteristics of hemangiosarcoma-associated skeletal muscle metastases in 61 dogs: A whole-body computed tomographic study. J. Vet. Int. Med., 33: 812-819. DOI: $10.1111 /$ jvim. 15456

Crawshaw, J., J. Berg, J.C. Sardinas, S.J. Engler and W.M. Rand et al., 1998. Henderson. Prognosis for dogs with nonlymphomatous, small intestinal tumors treated by surgical excision. J. Am. Anim. Hospital Assoc., 34: 451-6.

DOI: $10.5326 / 15473317-34-6-451$

Cruz-Arámbulo, R., R. Wrigley and B. Powers, 2004. Sonographic features of histiocytic neoplasms in the canine abdomen. Vet. Radiol. Ultrasound, 45: 554-558. DOI: $10.1111 /$ j.1740-8261.2004.04095.x

Culp, W.T., N. Ehrhart, S.J. Withrow, R.B. Rebhun and S. Boston et al., 2008. Results of surgical excision and evaluation of factors associated with survival time in dogs with lingual neoplasia: 97 cases (19952008). J. Am. Vet. Med. Assoc., 242: 1392-1397. DOI: $10.2460 /$ javma.242.10.1392

Daniaux, L.A., M.P. Laurenson, S.L. Marks, P.F. Moore and S.L. Taylor et al., 2013. Ultrasonographic thickening of the muscularis propria in feline small intestinal small cell T-cell lymphoma and inflammatory bowel disease. J. Feline Med. Surgery, 16: 89-89. DOI:10.1177/1098612X13498596

Fife, W.D., V.F. Samii, W.T. Drost, J.S. Mattoon and S. Hoshaw-Woodard, 2004. Comparison between malignant and non-malignant splenic masses in dogs using contrast-enhanced computed tomography. Vet. Radiol. Ultrasound, 45: 289-297. DOI: $10.1111 /$ j.1740-8261.2004.04054.X

Fondacaro, J.V., K.P. Richter, J.L. Carpenter, J.R. Hart and S.L. Hill et al., 1999. Feline gastrointestinal lymphoma: 67 cases. Eur. J. Comparative Gastroenterol., 4: 69-74.

Forrest, L.J., 2016. Computed tomography imaging in oncology. Vet. Clin. North Am. Small Anim. Practice, 46: 499-513. DOI: 10.1016/j.cvsm.2015.12.007

Frances, M., A.E. Lane and Z.M. Lenard, 2013. Sonographic features of gastrointestinal lymphoma in 15 dogs. J. Small Anim. Pract., 54: 468-474. DOI: $10.1111 /$ jsap. 12117

Fukushima, K., H. Kanemoto, K. Ohno, M. Takahashi and K. Nakashima et al., 2012. CT characteristics of primary hepatic mass lesions in dogs. Vet. Radiol. Ultrasound, 53: 252-257.

DOI: 10.1111/j.1740-8261.2011.01917.x

Fukushima, K., R. Fujiwara, K. Yamamoto, H. Kanemoto and K. Ohno et al., 2016. Characterization of triplephase computed tomography in dogs with pancreatic insulinoma. J. Vet. Med. Sci., 77: 1549-1553.I DOI: 10.1292/jvms.15-0077 
Gaschen, L., P. Kircher and J. Lang, 2003. Endoscopic ultrasound instrumentation, applications in humans and potential veterinary applications. Vet. Radiol. Ultrasound, 44: 665-680.

DOI: $10.1111 / \mathrm{j} .1740-8261.2003 . t b 00530 . x$

Gaschen, L., 2011. Ultrasonography of small intestinal inflammatory and neoplastic diseases in dogs and cats. Vet. Clin. North Am. Small Anim. Practice, 41: 329-344.

DOI: $10.1016 /$ j.cvsm.2011.01.002

Gaschen, L., P. Kircher and K. Wolfram, 2007. Endoscopic ultrasound of the canine abdomen. Vet. Radiol. Ultrasound, 48: 338-349. DOI: 10.1111/j.1740-8261.2007.00254.x

Gianella, P., M. Pietra, P.E. Crisi, P. Famigli Bergamini and F. Fracassi et al., 2017. Evaluation of clinicopathological features in cats with chronic gastrointestinal signs. Polish J. Vet. Sci., 20: 403-410. DOI: 10.1515/pjvs-2017-0052

Gieger, T., 2011. Alimentary lymphoma in cats and dogs. Vet. Clin. Small Anim., 41: 419-432.

DOI: $10.1016 /$ j.cvsm.2011.02.001

Griffin, S., 2019. Feline abdominal ultrasonography: What's normal? What's abnormal? The diseased gastrointestinal tract. J. Feline Med. Surgery, 21: 1047-1060. DOI: 10.1177/1098612X19880434

Gualtieri, M., M.G. Monzeglio and E. Scanziani, 1999. Gastric neoplasia. Vet. Clin. North Am. Small Anim. Practice, 29: 415-40. PMID: 10202797

Hobbs, J., D. Penninck and J. Lyons, 2015. Malignant transformation of a duodenal duplication cyst in a cat. J. Feline Med. Surgery.

Hoey, S., R. Drees and S. Hetzel, 2012. Evaluation of the gastrointestinal tract in dogs using computed tomography. Vet. Radiol. Ultrasound, 54: 25-30. DOI: 10.1111/j.1740-8261.2012.01969.x

Horvat, N., I. Petkovska and M.J. Gollub, 2018. MR Imaging of Rectal Cancer. Radiol. Clin. North Am., 56: 751-774. DOI: 10.1016/j.rcl.2018.04.004

Kaser-Hotz, B., B. Hauser and P. Arnold, 1996. Ultrasonographic findings in canine gastric neoplasia in 13 patients. Vet. Radiol. Ultrasound, 37: $51-56$

DOI: 10.1111/j.1740-8261.1996.tb00812.x

Kim, J.S., S.H. Park, S. Hansel and J.G. Fletcher, 2107. Imaging and screening of cancer of the small bowel. Radiol. Clin. North Am., 55: 1273-1291.

DOI: 10.1016/j.rcl.2017.06.008

Kutara, K., M. Seki, C. Ishikawa, M. Sakai and Y. Kagawa et al., 2014. Triple-phase helical computed tomography in dogs with hepatic masses. Vet. Radiol. Ultrasound, 55: 7-15.

DOI: $10.1111 /$ vru. 12099
Lamb, C.R. and J. Grierson, 1999. Ultrasonographic appearance of primary gastric neoplasia in $21 \mathrm{dogs}$. J. Small Anim. Practice, 40: 211-215. DOI: 10.1111/j.1748-5827.1999.tb03062.x

Larson, M.M. and D.S. Biller, 2009. Ultrasound of the gastrointestinal tract. Vet. Clin. Small Anim., 39: 747-759. DOI: 10.1016/j.cvsm.2009.04.010

Laurenson, M.P., K.A. Skorupski, P.F. Moore and A.L. Zwingenberger, 2011. Ultrasonography of intestinal mast cell tumors in the cat. Vet. Radiol. Ultrasound, 52: 330-4. DOI: 10.1111/j.1740-8261.2010.01792.x

Lingard, A.E., K. Briscoe, J.A. Beatty, A.S. Moore and A.M. Crowley et al., 2009. Low-grade alimentary lymphoma: Clinicopathological findings and response to treatment in 17 cases. J. Feline Med. Surgery, 11: 692-700.

DOI: $10.1016 /$ j.jfms.2009.05.021

Lubbes, D., P.J. Mandigers, H.C. Heuven and E. Teske, 2009. [Incidence of gastric carcinoma in Dutch Tervueren shepherd dogs born between 1991 and 2002]. Tijdschr Diergeneeskd, 134: 606-10.

PMID: 19697677

McLaughlin, P.D., K.P. Murphy, L. Crush, O.J. O'Connor and J.P. Coyle et al., 2013. Computed tomography colonography technique: The role of intracolonic gas volume. Radiol. Res. Practice.

Miyabayashi, T., J.P. Morgan, M.A.O. Atilola and L. Muhumuza, 1986. Small intestinal emptying time in normal beagle dogs. Vet. Radiol., 27: 164-168.

DOI: 10.1111/j.1740-8261.1986.tb00027.x

Morgan, J.P., 1981. The upper gastrointestinal examination in the cat: Normal radiographic appearance using positive contrast medium. Vet. Radiol., 22: 159-169.

DOI: 10.1111/j.1740-8261.1981.tb01367.x

Myers, N.C. and D.G. Penninck, 1994. Ultrasonographic diagnosis of gastrointestinal smooth muscle tumors in the dog. Vet. Radiol. Ultrasound, 35: 391-397. DOI: 10.1111/j.1740-8261.1994.tb02059.x

Newell, S.M., J.P. Graham, G.D. Roberts, P.E. Ginn and J.M. Harrison, 1999. Sonography of the normal feline gastrointestinal tract. Vet. Radiol. Ultrasound, 40: 40-43.

DOI: 10.1111/j.1740-8261.1999.tb01837.x

Nisa, K., S.Y. Lim, M. Shinohara, T. Osuga and N. Yokoyama et al., 2019. Evaluation of duodenal perfusion by contrast-enhanced ultrasonography in dogs with chronic inflammatory enteropathy and intestinal lymphoma. J. Vet. Int. Med., 33: 559-568. DOI: 10.1111 /jvim. 15432

Nyland, T.G., D.A. Neelis and J.S. Matoon, 2016. Gastrointestinal Tract. In: Small Animal Diagnostic Ultrasound, Nyland, T.G. and J.S. Mattoon (Eds.), Saunders Company, Philadelphia, pp: 485-518 
Paoloni, M.C., D.G. Penninck and A.S. Moore, 2002. Ultrasonographic and clinicopathologic findings in 21 dogs with intestinal adenocarcinoma. Vet. Radiol. Ultrasound, 43: 562-7. DOI: 10.1111/j.1740-8261.2002.tb01050.x

Paulin, M.V., L. Couronné, J. Beguin, S. Le Poder and M. Delverdier et al., 2018. Feline low-grade alimentary lymphoma: An emerging entity and a potential animal model for human disease. BMC Vet. Res., 14: 306-306. DOI: $10.1186 / \mathrm{S} 12917-018-1635-5$

Penninck, D.G., 1998. Characterization of gastrointestinal tumors. Vet. Clin. North Am. Small Anim. Practice, 28: 777-97.

DOI: 10.1016/s0195-5616(98)50078-2

Penninck, D.G., 2002. Gastrointestinal Tract. In: Small Animal Diagnostic Ultrasound, Nyland, T.G. and J.S. Mattoon (Eds.), Saunders Company, Philadelphia, pp: 207-230.

Penninck, D.G., A.S. Moore, A.S. Tidwell, M.E. Matz and G.O. Freden, 1994. Ultrasonography of alimentary lymphosarcoma in the cat. Vet. Radiol. Ultrasound, 35: 299-304.

DOI: 10.1111/j.1740-8261.1994.tb02045.x

Penninck, D.G., B. Smyers, C.R. Webster, W. Rand and A.S. Moore, 2003. Diagnostic value of ultrasonography in differentiating enteritis from intestinal neoplasia in dogs. Vet. Radiol. Ultrasound, 44: 570-575.

DOI: $10.1111 /$ j.1740-8261.2003.tb00509.x

Riedesel, E.A., 2017. Small Bowel. In: Textbook of Veterinary Diagnostic Radiology, Trhall, D.E. (Ed), Elsevier, St. Louis, pp: 926-954.

Rivers, B.J., P.A. Walter, D.A. Feeney and G.R. Johnston, 1997. Ultrasonographic features of intestinal adenocarcinoma in five cats. Vet. Radiol. Ultrasound, 38: 300-306.

DOI: $10.1111 /$ j.1740-8261.1997.tb00859.x

Russell, K., S. Mehler, K. Skorupski, J.L. Baez and F.S. Shofer et al., 2007. Clinical and immunohistochemical differentiation of gastrointestinal stromal tumors from leimyosarcomas leiomyosarcomas in dogs: 42 cases (1990-2003). J. Am. Vet. Med. Assoc., 230: 1329-33. DOI: 10.2460/javma.230.9.1329

Scanziani, E., A.M. Giusti, M. Gualtieri and D. Fonda, 1991. Gastric carcinoma in the Belgian shepherd dog. J. Small Anim. Practice, 32: 465-469. DOI: 10.1111/j.1748-5827.1991.tb00991.x

Seiler, G. and W. Maï, 2009. The Stomach. In: Bsava Manual of Canine and Feline Abdominal Imaging, O'Brien, R. and F. Barr (Eds.), British Small Animal Veterinary Association, Gloucester, pp: 87-109.
Slawienski, M.J., G.E. Mauldin, G.N. Mauldin and A.K. Patnaik, 1997. Malignant colonic neoplasia in cats: 46 cases (1990-1996). J. Am. Vet. Med. Assoc., 211: 878-81.

Stein, T.J., M. Pellin, H. Steinberg and R.T. Chun, 2010. Treatment of feline gastrointestinal small-cell lymphoma with chlorambucil and glucocorticoids. J. Am. Anim. Hosp. Assoc., 46: 413-417. DOI: $10.5326 / 0460413$

Swann, H.M. and D.E. Holt, 2002. Canine gastric adenocarcinoma and leiomyosarcoma: A retrospective study of 21 cases (1986-1999) and literature review. J. Am. Vet. Med. Assoc., 38: 157-164. DOI: 10.5326/0380157

Tanaka, T., H. Akiyoshi, K. Mie, M. Okamoto and Y. Yoshida et al., 2019. Contrast-enhanced computed tomography may be helpful for characterizing and staging canine gastric tumors. Vet. Radiol. Ultrasound, 60: 7-18. DOI: 10.1111/vru.12677

Terragni, R., A. Casadei Gardini, S. Sabattini, G. Bettini and D. Amadori et al., 2014a. EGFR, HER-2 and KRAS in canine gastric epithelial tumors: A potential human model? PLoS One, 9: e85388-e85388. DOI: 10.1371/journal.pone.0085388

Terragni, R., M. Vignoli, H.J. van Bree, L. Gaschen and J.H. Saunders, 2014b. Diagnostic imaging and endoscopic finding in dogs and cats with gastric tumors: A review. Schweiz Arch Tierheilk, 156: 569-576. DOI: 10.1024/0036-7281/a000652

Terragni, R., L. Morselli, M. Vignoli, E. Bottero and B. Brunetti et al., 2016. Serum Total LDH evaluation able to differentiate between alimentary lymphoma and inflammatory bowel disease in a real-world clinical setting? PLoS One, 11: e0151641-e0151641. DOI: 10.1371/journal.pone.0151641

Terragni, R., M. Vignoli, F. Rossi, P. Laganga and V.F. Leone et al., 2012. Stomach wall evaluation using helical hydro-computed tomography. Vet. Radiol. Ultrasound, 53: 402-405.

DOI: $10.1111 / \mathrm{j} .1740-8261.2012 .01928 . \mathrm{x}$

Teshima, T., H. Matsumoto, M. Michishita, K. Takahashi and H. Koyama, 2013. Multiple inflammatory gastric polyps treated by endoscopic polypectomy with argon plasma coagulation in a dog. J. Small Anim. Practice, 54: 265-268.

DOI: $10.1111 /$ jsap. 12032

Valentini, S., R. Tamburro, M. Zanatta, B. Brunetti and M. Gruarin et al., 2013. Intra-abdominal actinomycetoma in a dog caused by Actinomyces hordeovulneris. Pak. Vet. J., 34: 133-136.

Wallack, S.T., 2003. The Handbook of Veterinary Contrast Radiography. 1st Edn., San Diego Veterinary Imaging, Incorporated, ISBN-10: 0974344710, pp: 150. 
Willard, M.D., 2012. Alimentary neoplasia in geriatric dogs and cats. Vet. Clin. North Am. Small Anim. Practice, 42: 693-706. DOI: 10.1016/j.cvsm.2012.04.006

Withrow, S.J., 2007. Cancer of the Gastrointestinal Tract. In: Withrow and MacEwen's Small Animal Clinical Oncology, Withrow, S.J. and D.M. Vail (Eds.), Elsevier Saunders, St. Louis, pp: 455-510.

Yoon, S., S.K. Lee, J. Lee, Y.B. Baek and K. Cho et al., 2019. Dual-phase computed tomography angiography of intestinal carcinoid tumor as a lead point for cecocolic intussusception in a dog. J. Vet. Med. Sci., 81: 928-932. DOI: 10.1292/jvms.19-0101
Zwingenberger, A.L., S.L. Marks, T.W. Baker and P.F. Moore, 2010. Ultrasonographic evaluation of the muscularis propria in cats with diffuse small intestinal lymphoma or inflammatory bowel disease. J. Vet. Int. Med., 24: 289-292.

DOI: $10.1111 / \mathrm{j} .1939-1676.2009 .0457 . x$ 